\title{
A MAUT Approach for Reusing Ontologies
}

\author{
Antonio Jiménez $^{\# 1}$, Mari Carmen Suárez-Figueroa ${ }^{\# 2}$, Alfonso Mateos ${ }^{\# 3}$, Mariano Fernández-López ${ }^{* 4}$, Asunción \\ Gómez-Pérez ${ }^{\# 5}$ \\ ${ }^{\#}$ Departamento de Inteligencia Artificial, Universidad Politécnica de Madrid \\ Campus de Montegancedo S/N, Boadilla del Monte, 28660, Madrid, Spain \\ 1ajimenez@fi.upm.es \\ ${ }^{2}$ mcsuarezefi.upm.es \\ ${ }^{3}$ amateosefi.upm.es \\ ${ }^{5}$ agomez afi. upm. es \\ "Escuela Politécnica Superior, Universidad San Pablo CEU \\ Carretera de Boadilla del Monte km5.3, Boadilla del Monte, Spain \\ ${ }^{4}$ mfernandez .eps@ceu.es
}

\begin{abstract}
Knowledge resource reuse has become a popular approach within the ontology engineering field, mainly because it can speed up the ontology development process, saving time and money and promoting the application of good practices. The $\mathrm{NeOn}$ Methodology provides guidelines for reuse. These guidelines include the selection of the most appropriate knowledge resources for reuse in ontology development. This is a complex decision-making problem where different conflicting objectives, like the reuse cost, understandability, integration workload and reliability, have to be taken into account simultaneously.

GMAA is a PC-based decision support system based on an additive multi-attribute utility model that is intended to allay the operational difficulties involved in the Decision Analysis methodology. The paper illustrates how it can be applied to select multimedia ontologies for reuse to develop a new ontology in the multimedia domain. It also demonstrates that the sensitivity analyses provided by GMAA are useful tools for making a final recommendation.
\end{abstract}

\section{INTRODUCTION}

Knowledge has become increasingly important for supporting intelligent process automation and collaborative problem solving. The effective management of knowledge and shared data is currently one of the major challenges of large public and private organizations today.

Organizations manage crucial data and knowledge, that is, data about products, financial information, etc., that are recorded with the aim of supporting day-to-day company applications and decision-making processes.

Additionally, data and knowledge-management systems have evolved from centralized to decentralized systems as a result of an increase in the demand for resource sharing across different networked sites. Nonetheless, the key resource of current decentralized systems is still data and knowledge, and this means handling very large quantities of data and knowledge stored in different types of distributed archives. These data are normally structured, but sometimes they are very heterogeneous and, in most cases, not necessarily interoperable.

Nowadays, ontologies are used to specify the meaning of information for sharing. A solution for improving data and knowledge management is to set up an ontology-based application that properly manages all the organization's knowledge. This process involves modeling all this knowledge in an ontology network.

A great many ontologies have now been developed by different groups, taking different approaches and using different methods and techniques ([1]). The process of building ontologies from scratch is time and cost consuming.

One way of reducing the time and costs associated with the ontology development is by reusing available knowledge resources (ontologies, non-ontological resources, and ontology design patterns). Ontologies developed by reuse are also expected to spread good practices and increase the overall quality of ontological models.

With the goal of speeding up the ontology development process, ontology practitioners are starting, whenever possible, to reuse ([2]): (a) other ontologies such as SUMO (Suggested Upper Merged Ontology, which considers high level distinctions and contains temporal concepts and processes [3]), DOLCE (Descriptive Ontology for Linguistic and Cognitive Engineering, which is an ontology of particulars. http://www.loa-cnr.it/DOLCE.html), and KOWIEN (skill ontology from the university of Essen, which defines concepts representing the competencies required to describe job position requirements and job applicant skills. http://www. kowien.uni-essen.de/publikationen/konstruktion.pdf) and ontology modules ([4]); (b) ontology statements and ontology design patterns (ODPs) ([5], [6]); and (c) non-ontological resources ([7]), such as thesauri, lexicons, data bases, UML diagrams and classification schemas, such as NAICS (North American Industry Classification System, which provides industry-sector definitions for Canada, Mexico, and the United States to facilitate uniform economic studies across the boundaries of these countries. http://www.census.gov/epcd/ www/naics.html) and SOC (Standard Occupational Classification, which classifies workers into occupational categories: 23 major groups, 96 minor groups, and 449 occupations. http://www.bls.gov/soc/), built by other professionals and which have already gained some acceptance.

To support the ontology reuse process, the $\mathrm{NeOn}$ 
Methodology ([8]) provides some prescriptive methodological guidelines. These guidelines consist of the following activities: (1) search for candidate ontologies that could satisfy the needs of the ontology network being developed; (2) assess whether the set of candidate ontologies are useful for the development of the ontology network; (3) select the most suitable candidate ontologies for the development of the ontology network; and finally (4) integrate the selected ontologies into the ontology network being developed.

In this paper we focus on the third activity, that is, the selection of the most suitable ontologies for reuse in the development of a new ontology. This selection is a complex decision-making problem where different conflicting objectives, like the reuse cost, understandability, integration workload and reliability, have to be taken into account simultaneously. A subset of the candidate ontologies has to be selected. This subset should cover a high number of competency questions identified for the ontology being developed.

The Generic Multi-Attribute Analysis System (GMAA, http://www.dia.fi.upm.es/ ajimenez/GMAA) is a PC-based decision support system based on a multi-attribute additive utility model ([9], [10]) that is intended to allay the operational difficulties involved in the decision analysis (DA) methodology ([11]-[14]). GMAA is based on Multi-Attribute Utiliy Theory (MAUT) and accounts for uncertainty about the alternative performances and for incomplete information about decision-maker (DM) preferences, leading to classes of utility functions and weight intervals.

Throughout this paper we will illustrate the utilization of the GMAA system for selecting multimedia (MM) ontologies for reuse in the development of a new ontology, called $M 3$. This ontology should cover three perspectives: multimedia, multidomain and multilingual.

The paper is structured according to the steps of the DA cycle. Section 2 structures the problem. An objective hierarchy is built, attributes are established for the lowestlevel objectives and the candidate $\mathrm{MM}$ ontologies are identified. Section 3 quantifies the DM preferences. In Section 4 we evaluate the MM ontologies on the basis of a multiattribute additive utility function. Section 5 describes the sensitivity analyses provided by the GMAA system and demonstrates that they are useful for gaining further insight into the selection. Finally, some conclusions are provided in Section 6 .

\section{StRucturing THE PRoBlem}

First, we have to build an objective hierarchy, including all the relevant problem-related aspects. This hierarchy should be structured with objectives of similar importance at the same level to improve DM understanding of the decision.

Several conflicting objectives have to be taken into account simultaneously when selecting MM ontologies for reuse. The set of criteria described in [8] was adapted to the multimedia domain taking into account [15]. This led to 14 criteria organized according to four main objectives, as shown in Fig. 1.
Reuse cost is an estimate of the cost of reusing the candidate ontology. We considered the financial cost of reuse (Financ. Cost), an estimate of the economic cost needed for accessing and using the candidate ontology; and the required time for reuse (RequiredTime), which is the time it takes to access it.

Understandability (Understandab) is an estimate of the effort it takes to understand the candidate ontology. We consider the documentation quality, the availability of external knowledge and code clarity. Documentation quality (Doc Quality) refers to whether there is any communicable material explaining different aspects of the candidate ontology (e.g. modeling decisions). For instance, if there is a wiki, article or web page describing the candidate ontology, then it is assumed to be of high quality. The availability of external knowledge (Ext Knowledg) refers to whether the candidate ontology includes references to documentation sources and/or experts are easily available. Code clarity involves checking that the code is ease to understand and modify, that is, if the knowledge entities follow unified patterns and are clear. This criterion also refers to whether the code is documented, i.e. whether it includes clear and coherent definitions and comments for the knowledge entities represented by the candidate ontology.

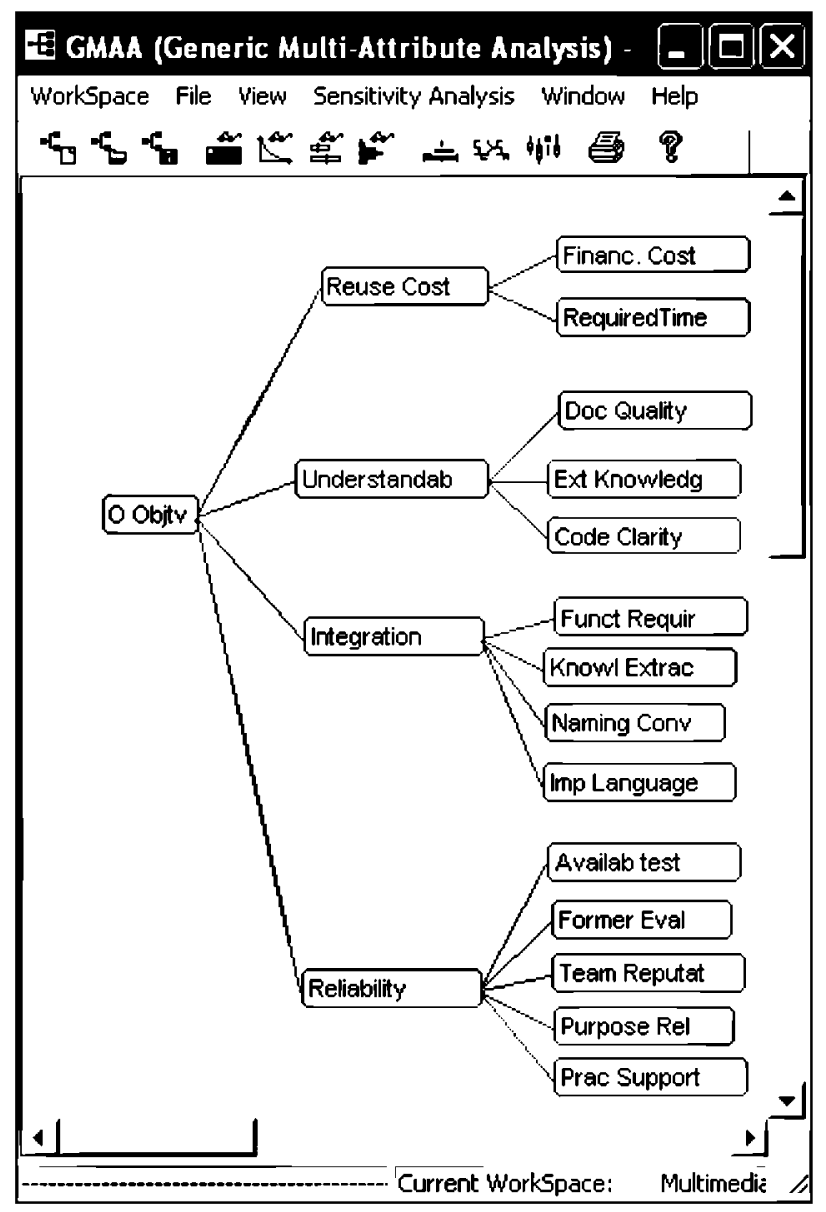

Fig. 1 Objective hierarchy 
Integration workload (Integration) is an estimate of the workload of integrating the candidate ontology into the ontology under development. We consider the number of functional requirements covered, the adequacy of knowledge extraction, the adequacy of naming conventions and the adequacy of the implementation language. The number of functional requirements covered (Funct Requir) refers to the number of competency questions (CQs) [16] covered by the ontology candidate. The adequacy of knowledge extraction (Knowl Extrac) checks whether it is easy to identify parts of the candidate ontology to be reused or extracted. The adequacy of naming conventions (Naming Conv) is set to low if the names are not intuitive, medium if they are clearly understandable and high if they are taken from a given standard (e.g. W3C, MPEG7, etc.). Finally, the adequacy of the implementation language (Imp Language) checks whether both languages (the candidate's ontology and the ontology's being developed) are the same or at least are able to represent similar knowledge with the same granularity.

Finally, reliability analyses whether the candidate ontology for reuse is trustworthy. Reliability is assessed according to availability of tests, development team reputation, purpose reliability and practical support. The availability of tests (Availab test) refers to whether tests are available for the candidate ontology for reuse. Former evaluation refers to whether the ontology has been properly evaluated, i.e. whether it has passed a set of unit tests. Development team reputation (Team Reputat) refers to whether the development team is reliable. Purpose reliability (Purpose Rel) accounts for academic use, transformed from standard metadata and developed in a project. Finally, practical support (Prac Support) checks whether well-known projects or ontologies have reused the candidate ontology (for instance, ontologies built within a project and using ontology design patterns score highest).

Next, attributes have to be established for the lowest-level objectives in the hierarchy. These attributes are used to indicate the extent to which the considered alternatives achieve the lowest-level objectives. Most criteria were assessed on a discrete scale. A fully detailed description of the criteria and how they are measured is available in [8]. For instance, for Adequacy of the implementation language the following values were considered: low; when the candidate ontology and the one being developed are not in the same language and there are no transformation mechanisms between both languages; medium, when they are not in the same language but there is an available mechanism to make the transformation and; high, when the language is the same in both ontologies. A fully detailed description of the criteria and how they are measured is available in [8].

The next step in the DA cycle is to identify candidate MM ontologies and their performances in terms of the attributes associated with the lowest-level objectives.

To identify the candidate MM ontologies we searched for MM ontologies that could satisfy the needs of the M3 ontology, particularly, the multimedia perspective, leading to 40 ontologies. Then, we carried out a deep study of these ontologies to analyze their scope, purpose, and functional and non-functional requirements regarding those established for the $M 3$ ontology. This led to 23 candidate ontologies ([15]), including the following: COMM, MPEG7 Hunter, MPEG-7x, SAPO, DIG35, CSO, AceMedia VDO, VRACORE3 ASSEM, Boemie VDO, Music Ontology, Media Ontology, Kanzaki Music, Music Ontology, MusicRights, Open Drama, MPEG7 MDS, VraCore3 Simile, Nokia Ontology, SRO, Device Ontology, MPEG7 Ontology, Photography Ontology and M3O.

The MM ontology performances for the established attributes of the lowest-level objectives are available in [15], see Fig 2.

Although the GMAA system accounts for uncertainty about alternative performance, the values entered originally were precise. We accounted for missing performances, i.e. the performance of at least one MM ontology was unknown for some criteria.

\section{QUANTIFYING PREFERENCES}

Quantifying preferences involves assessing DM single attribute utilities and weights. Attribute utilities represent DM preferences concerning the possible alternative performances, and weights represent the relative importance of criteria accounting for DM preferences.

In both cases, the system admits imprecise information through value intervals as responses to the probability questions that the DM is asked. This leads to classes of utility functions and weight intervals, respectively. This is less demanding for a single DM and also makes the system suitable for group decision support (e.g. [17]).

Imprecise component utilities were assigned for the respective discrete attributes, whereas a linear utility function was assigned for the number of functional requirements covered criterion, as shown in Fig. 3 .

Note that a utility equal to 1 corresponds to the best attribute performance, whereas a 0 utility is assigned to the least preferred performance. ValueT in Fig. 3 refers to the number of $C Q \mathrm{~s}$ covered, as mentioned in Section II. It is assessed as follows

$$
\text { ValueT }=\frac{\text { number of } C Q \text { s covered } \times M N V L T}{\text { total number of } C Q S},
$$

where number of $C Q$ s covered is the number of $C Q s$ identified for the $M 3$ ontology that are fulfilled by the candidate ontology, MNVLT is the maximum numerical value in linguistic transformation and is set to 3 , as established in [15]; and total number of $C Q s$ is the number of $C Q s$ identified for the $M 3$ ontology.

As mentioned before, we accounted for missing performances, i.e. if the performance of at least one MM ontology is unknown for a criterion, then an additional attribute value is considered, and the associated utility is the interval $[0,1]$, as justified in [18]. 


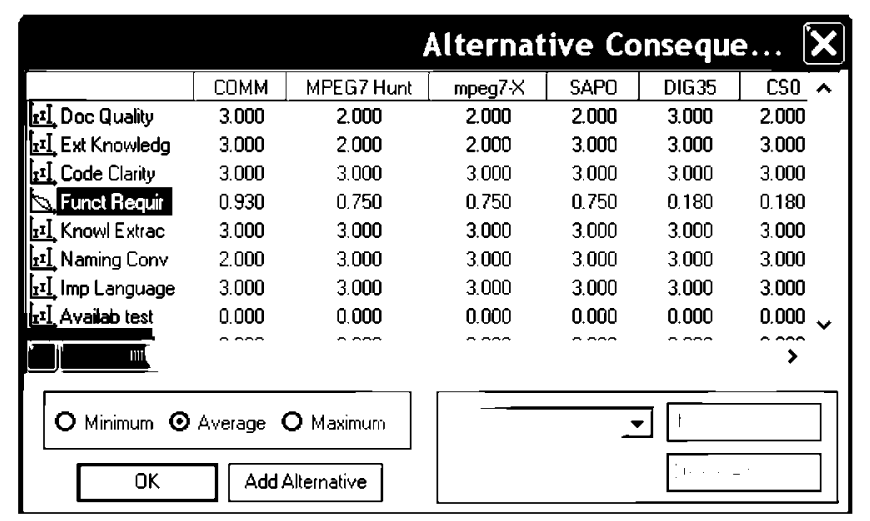

Fig. $2 \mathrm{MM}$ ontology performances

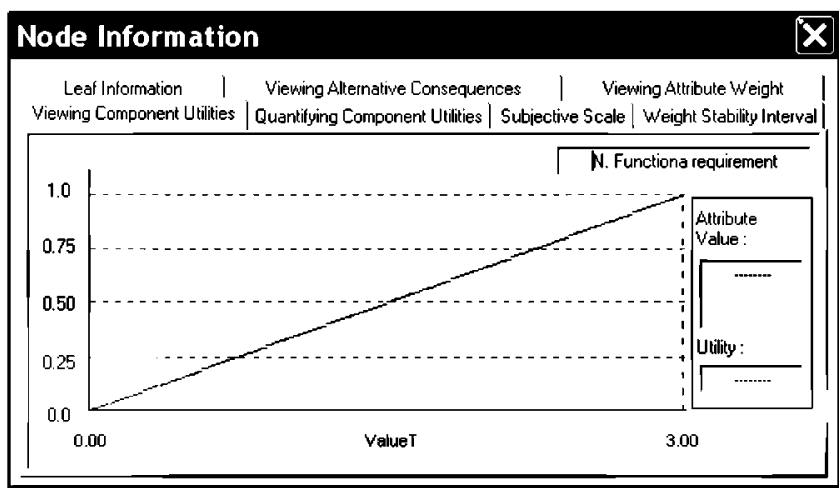

Fig. 3 Component utilities for number of functional requirements covered

Fig. 4 shows the discrete attribute values for the Purpose reliability criterion and the respective component utilities: 0 unknown, 1-low (an ontology built for academic use), 2medium (an ontology transformed from standard metadata by a reputed team) and 3-high (an ontology developed in a project).

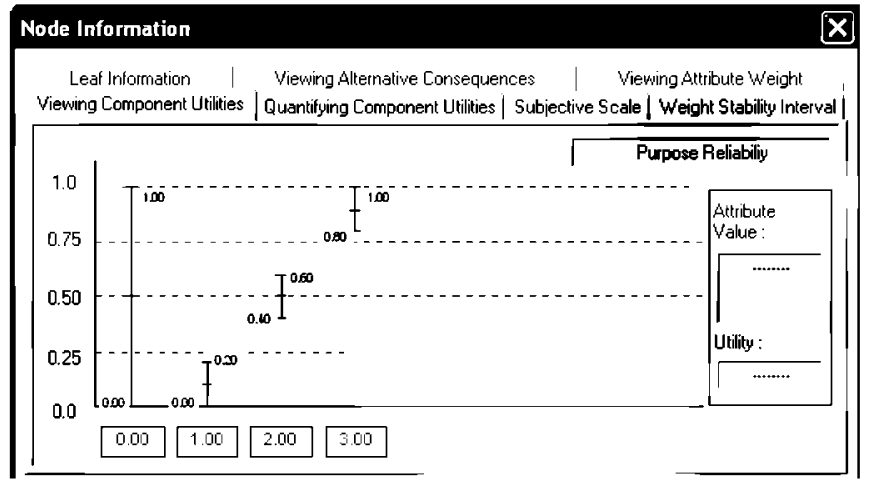

Fig. 4 Imprecise component utilities for Purpose reliability discrete values

Imprecise weights representing the relative importance of criteria are elicited along the branches of the hierarchy using a method based on trade-offs. Then the attribute weights used in the multi-attribute additive utility model are assessed by multiplying the elicited weights in the path from the overall objective to the respective attributes, see Fig. 5.

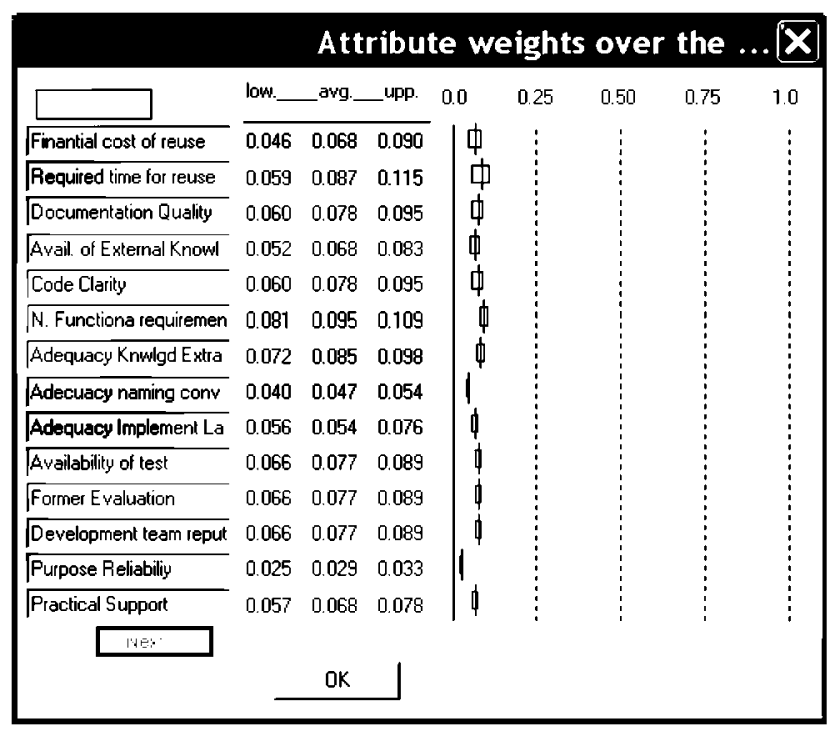

Fig. 5 Attribute weights in the additive multi-attribute utility model

The average normalized weight (avg.) and normalized weight intervals (bounded by low. and upp.) are shown in Fig. 5 for each of the 14 attributes established in the lowest-level objectives.

\section{EVALUATING MM ONTOLOGIES}

The different $\mathrm{MM}$ ontologies under consideration can be evaluated by means of a multi-attribute additive utility function

$$
u\left(O_{i}\right)=\sum_{j=1}^{n} w_{j} u_{i j}\left(x_{i j}\right),
$$

where $x_{i j}$ is the performance in attribute $X_{j}$ of MM ontology $O_{i} ; u_{j}\left(x_{i j}\right)$ is the utility associated with $x_{i j}$, and $w_{j}$ are the weights of each attribute.

The additive model is considered a valid approximation in most real decision-making problems for the reasons described in [19] and [20]. It is used to assess average overall utilities and minimum and maximum overall utilities. The ranking of MM ontologies is based on average overall utilities, and minimum and maximum overall utilities give further insight into the robustness of this ranking, as shown in Fig. 6

The ranking output by the GMAA system is very similar to the ranking in [15], where missing performances were not correctly modeled (worst attribute performances were assigned)

Based on the methodological guidelines for reusing ontologies included in the NeOn Methodology, a subset of the best-ranked ontologies (in this case in the multimedia domain) has to be selected. This subset should cover a high number of CQs identified for the ontology being developed.

The average utility for the three best-ranked alternatives is almost the same and the utility difference among the eight best-ranked candidates is less than 0.1 . As the output utility intervals are very overlapped, we concluded that this evaluation did not obtain meaningful enough information to make a final selection. Consequently, sensitivity analysis should be carried out to provide further insight into the subset of best ontologies for reuse. 


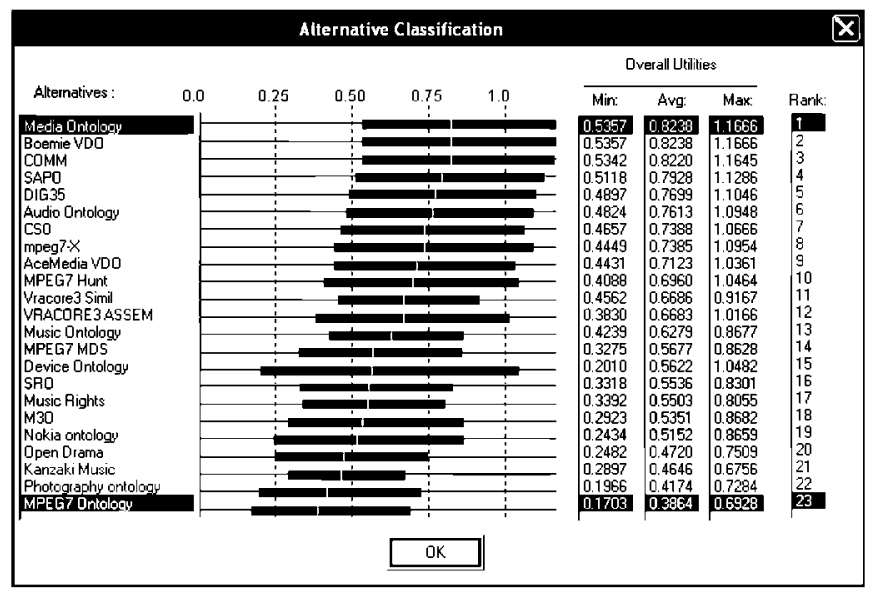

Fig. 6 Ranking of MM ontologies

In the GMAA system we can also select another objective to rank by. Fig. 7 shows the ranking of MM ontologies for Understandability, i.e. only the documentation quality, availability of external knowledge and code clarity attributes are evaluated.

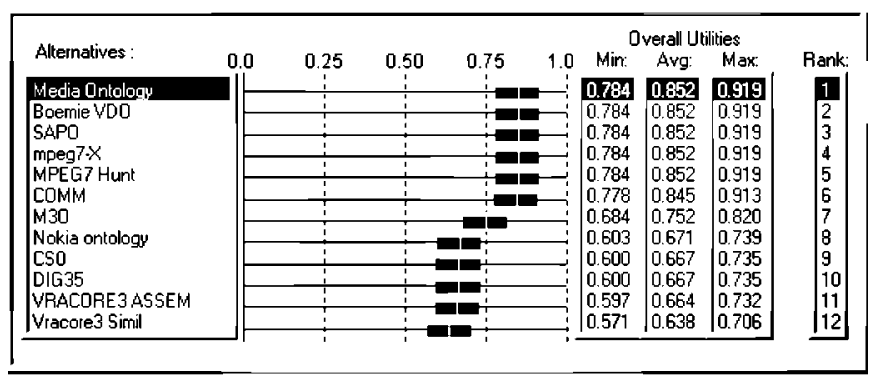

Fig. 7 Ranking for Understandability

\section{SENSITIVITY ANALYSIS}

The GMAA provides several types of Sensitivity Analysis (SA). It computes the stability weight interval for any objective at any level in the hierarchy. This represents the interval where the average normalized weight for the considered objective can vary without affecting the overall ranking of alternatives or just the best-ranked alternative. This SA can be useful for identifying weight objectives to which the $\mathrm{MM}$ ontologies ranking is sensitive.

Weights stability intervals were assessed regarding the best-ranked $\mathrm{MM}$ ontology and the interval $[0,1]$ was output for all the criteria at the different levels and branches of the hierarchy except for the number of functional requirements and the adequacy of naming conventions, see Fig. 8. This means that Media Ontology is still the best-ranked candidate whatever average normalized weights are assigned for practically all the objectives.

We considered that weight stability intervals were not meaningful for the overall ranking of candidates mainly because candidates were analyzed, and no information about rank reordering was provided.

The other two SAs provided by the GMAA system, the assessment of non-dominated and potentially optimal alternatives and the application of Monte Carlo simulation techniques, can be considered as decision making with partial information ([21], [22]). Both intend to take advantage of the imprecise information gathered during the assessment of component utilities and weights.

The GMAA system computes the potentially optimal alternatives among the non-dominated alternatives ([23]-[25]) because these are alternatives that are best ranked for at least one combination of the imprecise parameters, i.e., weights and component utility functions.

However, 20 out of the $23 \mathrm{MM}$ ontologies are nondominated and potentially optimal. As a result, this SA can only discard three MM ontologies (Kanzai Music, Photography Ontology and DIG35) and a further analysis is still required to make a final selection.

Using Monte Carlo simulation techniques simultaneous changes can be made to the weights and generate results that can be easily analyzed statistically to provide more insight into the multi-attribute model recommendations ([26], [27]).

Three general classes of simulation are possible in the GMAA system. First, the system can generate attribute weights completely at random (there is no knowledge whatsoever of the relative importance of the attributes). Another possibility is to randomly generate the attribute weights preserving a total or partial attribute rank order. Finally, attribute weights can be randomly assigned values taking into account the elicited weight intervals, see Fig 5

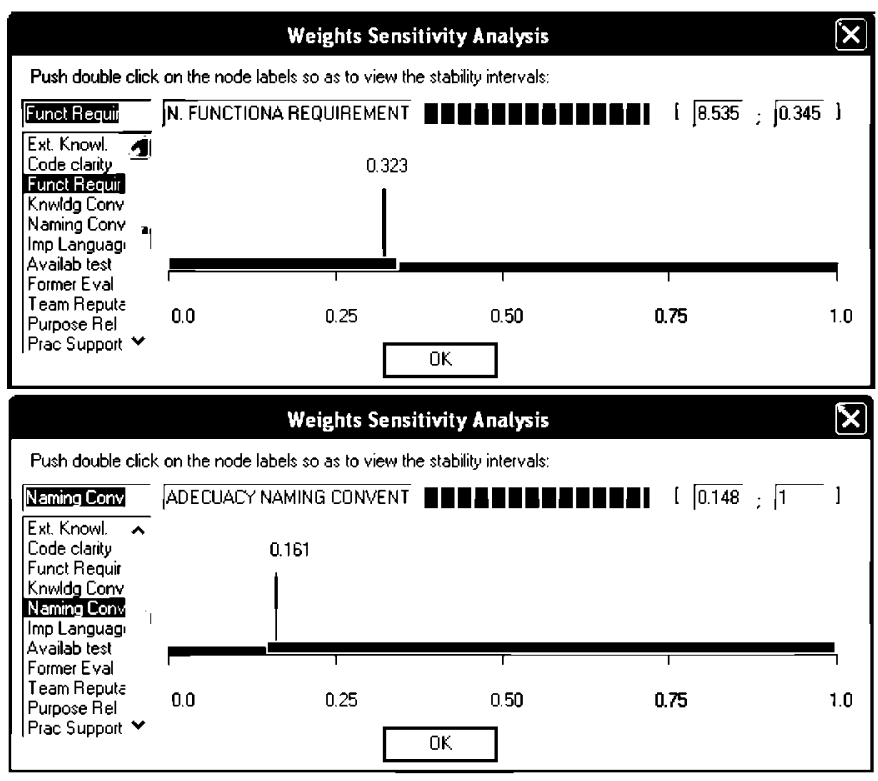

Fig. 8 Weight stability intervals

While the simulation is running, the system computes several statistics about the rankings of each MM ontology, like mode, minimum, maximum, mean, standard deviation and the 25th, 50th and 75th percentiles. This information can be useful for discarding some available alternatives, aided by a display that presents a multiple boxplot for the alternatives.

Fig. 9 shows the resulting multiple boxplot when the elicited weights intervals are used to randomly generate values (the respective statistics are shown in Fig. 10). 


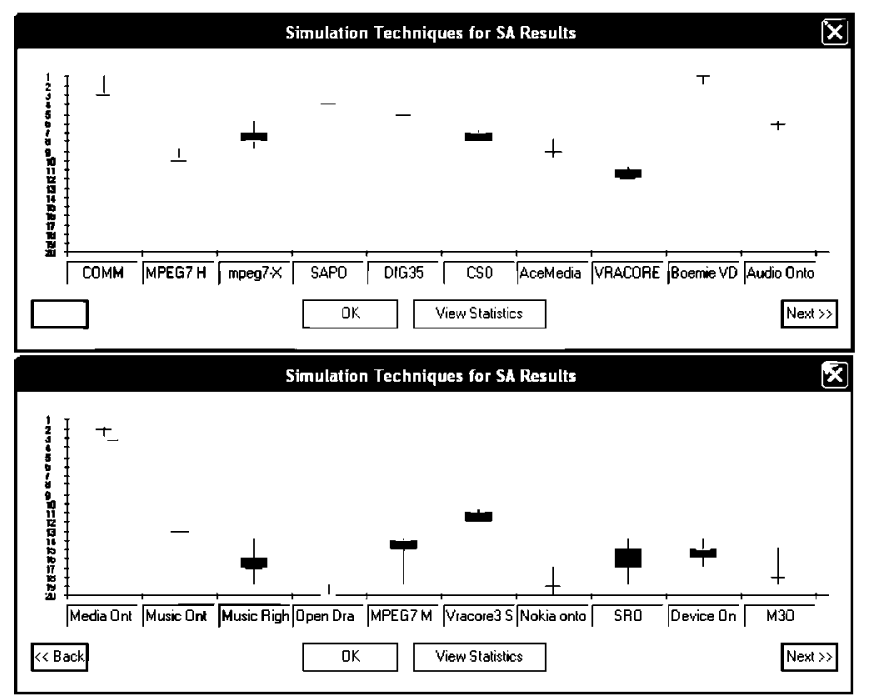

Fig. 9 Multiple boxplot

We have highlighted the boxplots for the best five MM ontologies: Media Ontology, Boemie VDO, COMM, SAPO and Music Ontology. They match up with the results of the average overall utilities since the five best-ranked $\mathrm{MM}$ ontologies are the same, as shown in Fig. 6. Only two MM ontologies -Media Ontology and Boemie VDO- were ranked best across all 10,000 simulations, which are the two best ranked candidates on the basis the average utilities.

Moreover, the rankings for the best five MM ontologies fluctuate by at most two positions throughout the simulation. Consequently, we can conclude that the ranking is robust.

Based on the methodological guidelines for reusing ontologies included in the $\mathrm{NeOn}$ Methodology, as the number of $C Q s$ covered by the five best-ranked MM ontologies was higher than $70 \%$, no more ontologies were necessary for reuse in the development of the $M 3$ ontology.

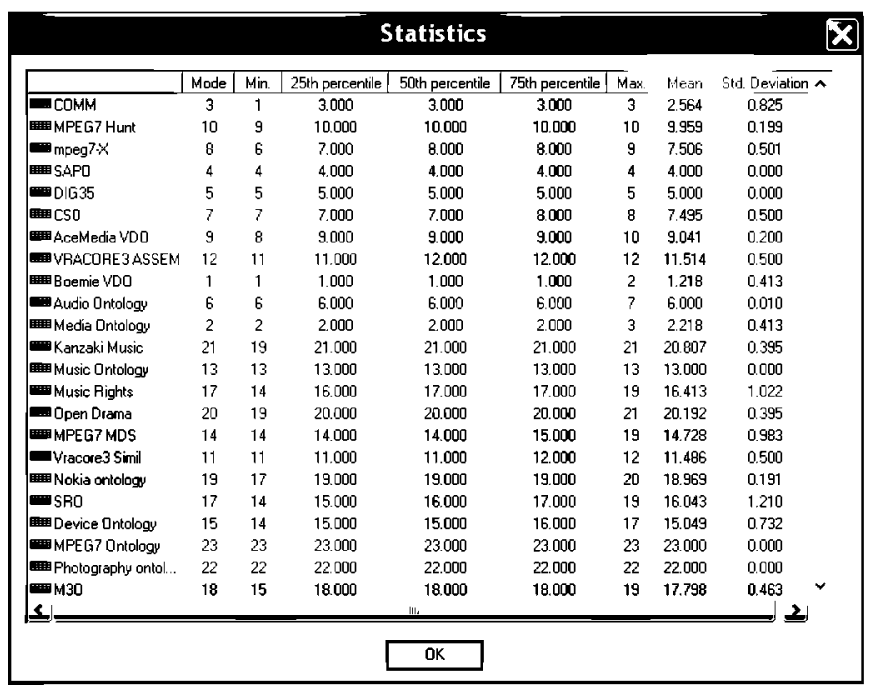

Fig. 10 Boxplot Statistics

\section{CONCLUSIONS}

The aim of the ontology reuse process is to find and select a set of ontologies useful for building a new ontology. The Neon Methodology provides prescriptive methodological guidelines to perform such reuse process. These guidelines consists of different activities, including the selection of the most appropriate ontologies for reuse, a complex decisionmaking problem including different conflicting objectives.

In this regards, the GMAA system has proved to be a useful tool for such a situation. It helps DMs to think about the problem in more depth, and accounts for imprecision in the inputs (ontology performances and DM's preferences). The provision for imprecision makes the process less stressful for experts and makes the system suitable for group decisionmaking, where individual conflicting views in a group of DMs can be captured through imprecise answers.

The system provides sensitivity analyses to give further insight into the robustness of the resulting ranking. Monte Carlo simulation techniques have been proved to be especially useful for exploiting imprecise information about the input parameters to achieve a final recommendation on the selection of $\mathrm{MM}$ ontologies for reuse.

\section{ACKNOWLEDGMENT}

The paper was supported by Madrid Regional Government project S-2009/ESP-1685, the Spanish Ministry of Education and Science project TIN 2008-06796-C04-02, the Spanish Ministry of Science and Innovation project MTM2011-28983C03-03 and the CENIT-E project BUSCAMEDIA (CEN2009-2016), co-funded by the Centro de Desarrollo Tecnológico Industrial (CDTI). We would like to thank our project partners for their help

\section{REFERENCES}

[1] A. Gómez-Pérez, M. Fernández-López and O. Corcho, Ontological Engineering. Berlin, Germany: Springer Verlag, 2003.

[2] E. Simperl, "Reusing ontologies on the Semantic Web: a feasibility study", Data Knowl Eng, vol. 68, pp. 905-925, 2009.

[3] R.A. Pease, I. Niles and J. Li, "The suggested upper merged ontology: a large ontology for the semantic web and its application", in Proc. of AAAI 2002, 2002.

[4] B. Cuenca-Grau, I. Horrocks, Y. Kazakov and U. Sattler, "Just the right amount: extracting modules from ontologies", in Proc. of 16 th Int. Conf. on WWW, pp. 717-726, 2007.

[5] A. Gangemi, "Design patterns for legal ontology construction", in Trends in legal knowledge: The semantic web and the regulation of electronic social systems. European Academic Publishing, 2007.

[6] V. Presutti and A. Gangemi, "Content ontology design patterns as practical building blocks for web ontologies", in Lecture Notes in Computer Science, vol. 5231, pp. 128-141. Heidelberg: Springer, 2008.

[7] A. Jimeno-Yepes, E. Jiménez-Ruíz, R. Berlanga-Llavori and D. Rebholz-Schuhmann, "Reuse of terminological resources for efficient ontological engineering in Life Sciences", BMC Bioinformatics, vol. 10 , pp. 1471-2105, 2009.

[8] M.C. Suárez-Figueroa, "NeOn methodology for building ontology networks: specification, scheduling and reuse", $\mathrm{PhD}$ thesis, Universidad Politécnica de Madrid, Madrid, Spain, 2010.

[9] A. Jiménez, S. Ríos-Insua, and A. Mateos, "A decision support system for multiattribute utility evaluation based on imprecise assignments", Decis Sup Syst, vol. 36, pp. 65-79, 2003.

[10] A. Jiménez, S. Ríos-Insua and A. Mateos, "A generic multi-attribute analysis system", Comput Oper Res, vol. 33, pp. 1081-1101, 2006. 
[11] V. Belton, "Multiple criteria decision analysis - Practically the only way to choose", in Operational research tutorial papers, Birmingham, UK: Operational Research Society, pp. 53-101, 1990.

[12] R.L. Keeney and H. Raiffa, Decisions with Multiple Objectives: Preferences and Value Tradeoffs, Cambridge, UK: Cambridge University Press, 1993.

[13] R.T. Clemen, Making Hard Decisions, Belmont, USA: Duxbury Press, 1996.

[14] C.W. Kirkwood, Strategic Decision Making: Multiobjective Decision Analysis with Spreadsheets, Beltmont, USA: Duxbury Press, 1997.

[15] G.A. Atemezing, "Analyzing and ranking multimedia ontologies for their reuse", M Eng thesis, Universidad Politécnica de Madrid, Madrid, Spain, 2010.

[16] M. Grüninger and M.S. Fox, "Methodology for the design and evaluation of ontologies", in Proc. of IJCAI95, pp 6.1-6.10, 1995.

[17] A. Jiménez, A. Mateos and S. Ríos-Insua, "Monte Carlo simulation techniques in a decision support system for group decision-making", Group Dec Negotiation, vol. 14, pp. 109-130, 2005.

[18] A. Jiménez, S. Ríos-Insua and A. Mateos, "Missing consequences in multi-attribute utility theory", OMEGA, vol. 37, pp. 395-410, 2009.

[19] H. Raiffa, The Art and Science of Negotiation. California, USA: Harvard University Press, 1982.
[20] T.J. Stewart, "Robustness of additive value function method in MCDM", J Multi-Criteria Dec Anal, vol. 5, pp. 301-309, 1996

[21] D. Ríos Insua, Sensitivity Analysis in Multi-objective Decision Making. New York, USA: Springer, 1990

[22] D. Ríos and S. French, "A framework for sensitivity analysis in discrete multi-objective decision-making", Eur J Oper Res, vol. 54, pp. 176-190, 1991.

[23] Y.S. Eum, K.S. Park and S.H. Kim, "Establishing dominance and potential optimality in multi-criteria analysis with imprecise weight and value", Comput Oper Res, vol. 28, pp. 397-409, 2001.

[24] K.S. Lee, K.S. Park and S. H. Kim, "Dominance, potential optimality, imprecise information and hierarchical structure in multicriteria analysis", Comput Oper Res, vol. 29, pp. 1267-81, 2002.

[25] A. Mateos, S. Ríos-Insua and A. Jiménez, "Dominance, potential optimality and alternative ranking in imprecise decision making", $J$ Oper Res Soc, vol. 58, pp. 326-336, 2007.

[26] A. Mateos, A. Jiménez and S. Ríos-Insua, "Monte Carlo simulation techniques for group decision-making with incomplete information". Eur J Oper Res, vol. 174, pp. 1842-1864, 2006.

[27] J. Butler, J. Jia and J. Dyer, "Simulation techniques for the sensitivity analysis of multi-criteria decision models", Eur J Oper Res, vol. 103, pp. 531-46, 1997. 